\title{
Bone Mineral Density and the Results of Chiari Pelvic Osteotomy for Osteoarthritis of the Hip
}

\author{
TOSHIAKI ASAKURA \\ Asakura Orthopaedic Clinic, Kurume 830, Japan
}

Received for publication June 16, 1997

\begin{abstract}
Summary: The aim of this study was to discover the involvement, if any, by bone mineral density (BMD) in the results after osteotomy for osteoarthritis of the hip (OA). The BMD, the Bombelli classification and the postoperative results in 36 joints after Chiari pelvic osteotomy for OA in the advanced and late stage were investigated for any correlations. The Bombelli hypertrophic type showed significantly better improvement than the atrophic type, but there was no correlation between the value of the BMD, and the postoperative result.
\end{abstract}

Key words bone mineral density, osteoarthritis of the hip, Chiari pelvic osteotomy, osteoblastic changes, biological response

\section{Introduction}

In osteoarthritis (OA) accompanying osteoblastic changes (such as involving osteophytes or sclerosis), the postoperative results after a joint-preserving operation or after total hip athroplasty (THA) are known to improve. However, the mechanism for their improvements are not yet understood. The aims of the present study were to investigate whether or not osteoblastic change in the hip joint between before and after the operation were related to the value of the bone mineral density (BMD) and to elucidate whether any osteoblastic change in the hip joint was localized to the hip joint or was systemic throughout the body.

\section{Subjects and Methods}

\section{Subjects}

Since it is in advanced and late stage
$\mathrm{OA}$ in young and adult patients that osteoblastic change become a subject of discussion, I examined 36 joints in 36 patients whose OA was in the advanced or late stage preoperatively, and followed these for more than 1 year postoperatively. Measurements of the BMD were taken before or after Chiari pelvic osteotomy. The patients involved 35 females and 1 male. Their age at operation ranged from 26 to 67 years (mean 51.3 years). The follow-up period ranged from 1 year to 8 years and 8 months, with a mean follow-up period of 3 years and 6 months.

\section{Operative method}

In modified Chiari pelvic osteotomy (Inoue et al. 1995), the patient is placed in the lateral position. Through a lateral approach, the greater trochanter undergoes osteotomy and the acetabular edge is reached. After the level and angle for osteotomy are ascertained by X-ray 
control, osteotomy of the pelvis is performed in dome shape along the acetabular edge. When femoral osteotomy is required, it is performed subsequently in a combined operation. After the desired displacement is acquired by manipulation under X-ray control, percutaneous fixation of the fragment is done using Kirschner-wires and the wound is closed.

\section{Evaluation method}

Evaluation of the operative results: The criteria for OA by the Japanese Orthopaedics Association (JOA) score was used for the evaluation of clinical results. According to these criteria, 40 points are given for no pain, 20 for range of motion, 20 for walking ability, and 20 for activities in daily living, with a total score of 100 . A score of 90 points or more was judged as excellent, that of 80 points or more and less than 90 as good, that of 70 points or more and less than 80 as fair, and that below 70 points poor.

Bone mineral density measurement: BMD was measured using dual photon X-ray absorptiometry (DPX) (LUNAR Radiation: Tokyo Corp.) and autoanalysis. Measurements were performed on the bilateral hip joints, total body, and the anteroposterior lumbar spine. The measured BMD was then compared to age-matched normal levels and \% agematched was calculated. Correlation with the clinical results was examined on individual values.

Preoperative typing: Using a preoperative X-ray anteroposterior view of the hip joint, the type of osteoarthritis was classified as hypertrophic type, normotrophic type or as atrophic type, according to Bombelli's classification based on biological response (1983). Briefly, a hypertrophic type has remarkable osteophyte is development and. a large capital drop is formed inferomedially to the femoral head. A normotrophic type has moderate osteophyte formation. And an atrophic type has almost no osteophyte formation, and the size of the femoral head is decreased in some cases.

Determination of the Bombelli classification was undertaken by 2 orthopaedic surgeons in a blind fashion. The BMD values were then compared with the obtained clinical results, for each Bombelli type. The Scheffe method was used for testing significance, and 5\% was taken as the level of significance.

\section{Results}

Under the Bombelli classification, 9 hips were hypertrophic type, 14 were normotorophic, and 13 hips were atrophic type. Of 36 patients, 26 (72.2\%) showed excellent or good results, with 80 points or more in the postoperative JOA score (Table 1). The change in JOA score between before and after the operation was called the degree of

TABLE 1.

Results after of Chiari pelvic osteotomy according to Bombelli classification

\begin{tabular}{cccccc}
\hline \multirow{2}{*}{ Bombelli } & \multirow{2}{*}{$\begin{array}{c}\text { No. of } \\
\text { Patients }\end{array}$} & \multicolumn{4}{c}{ Clinical Results } \\
\cline { 3 - 6 } & & Excellent & Good & Fair & Poor \\
\hline Hypertrophic & 9 & 5 & 2 & 2 & 0 \\
Normotrophic & 14 & 10 & 1 & 2 & 1 \\
Atrophic & 13 & 5 & 3 & 1 & 4 \\
\hline
\end{tabular}

Of 36 patients, 26 (72.2\%) showed excellent or good results. 


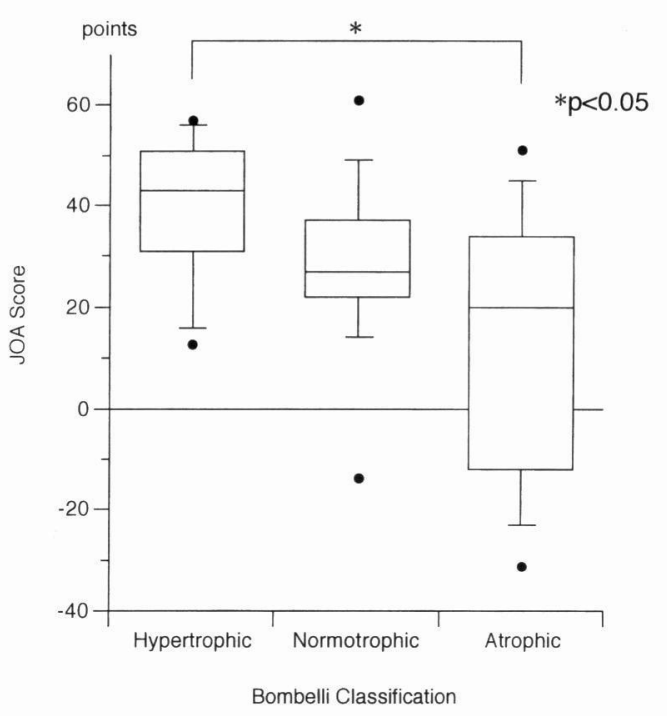

Correlation between each type of Bombelli classification and improvement in JOA score

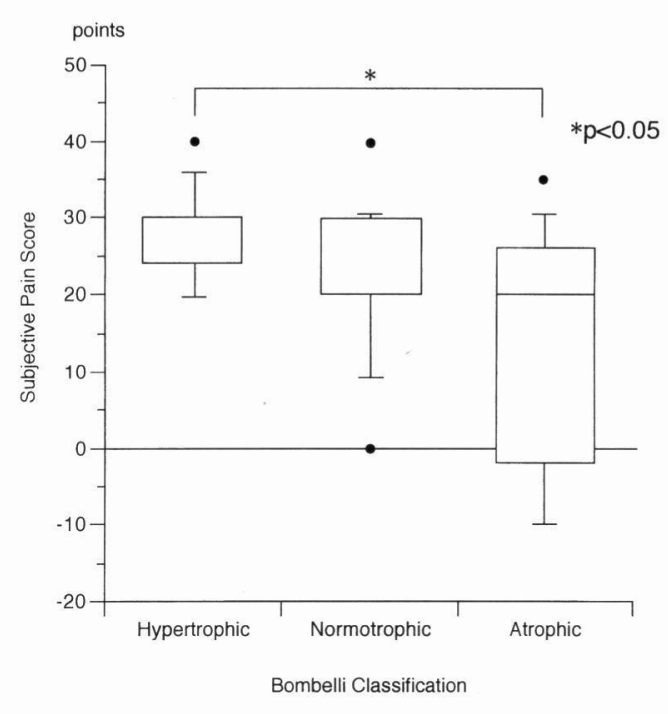

Correlation between each type of Bombelli classification and improvement in pain

Fig. 1.

Fig. 2.

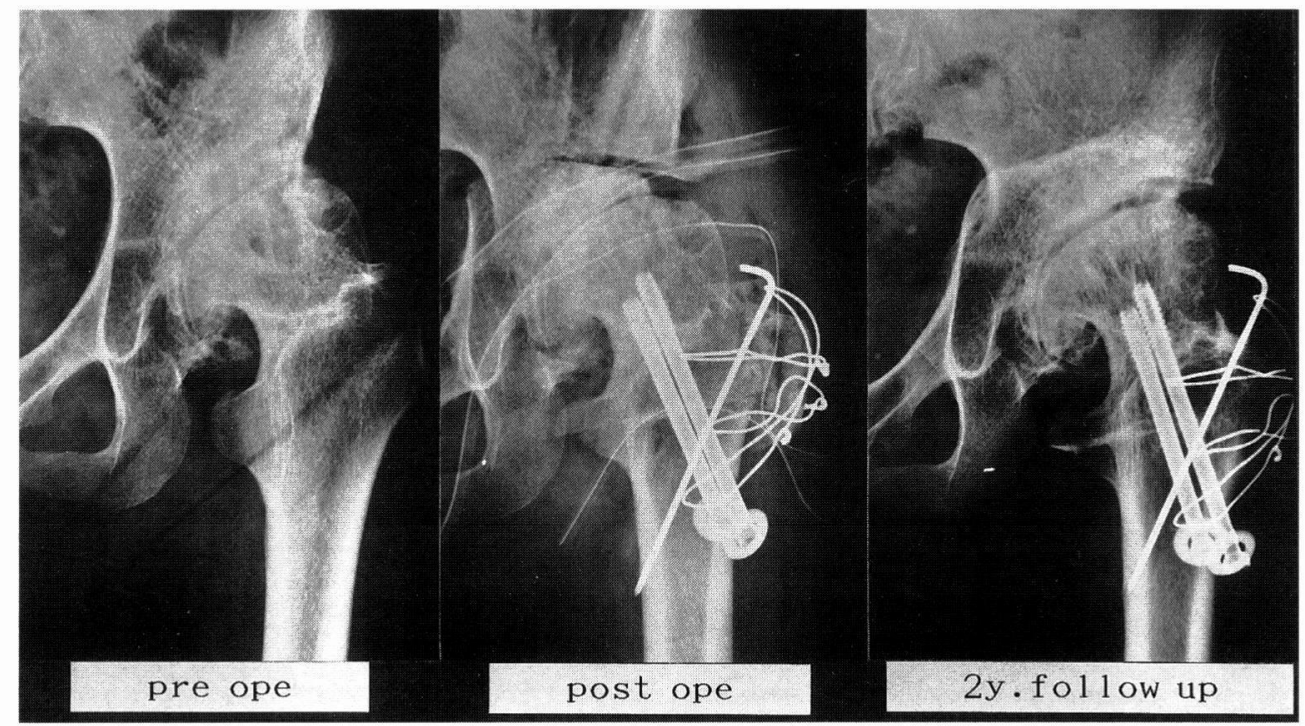

Fig. 3. Case 1. 45y. F. OA late stage, hypertrophic type: Neck $\%$ age matched $108 \%$. Chiari and valgus osteotomy. Postoperative JOA score improved from 34 points to 91 points at 2 years follow up. 


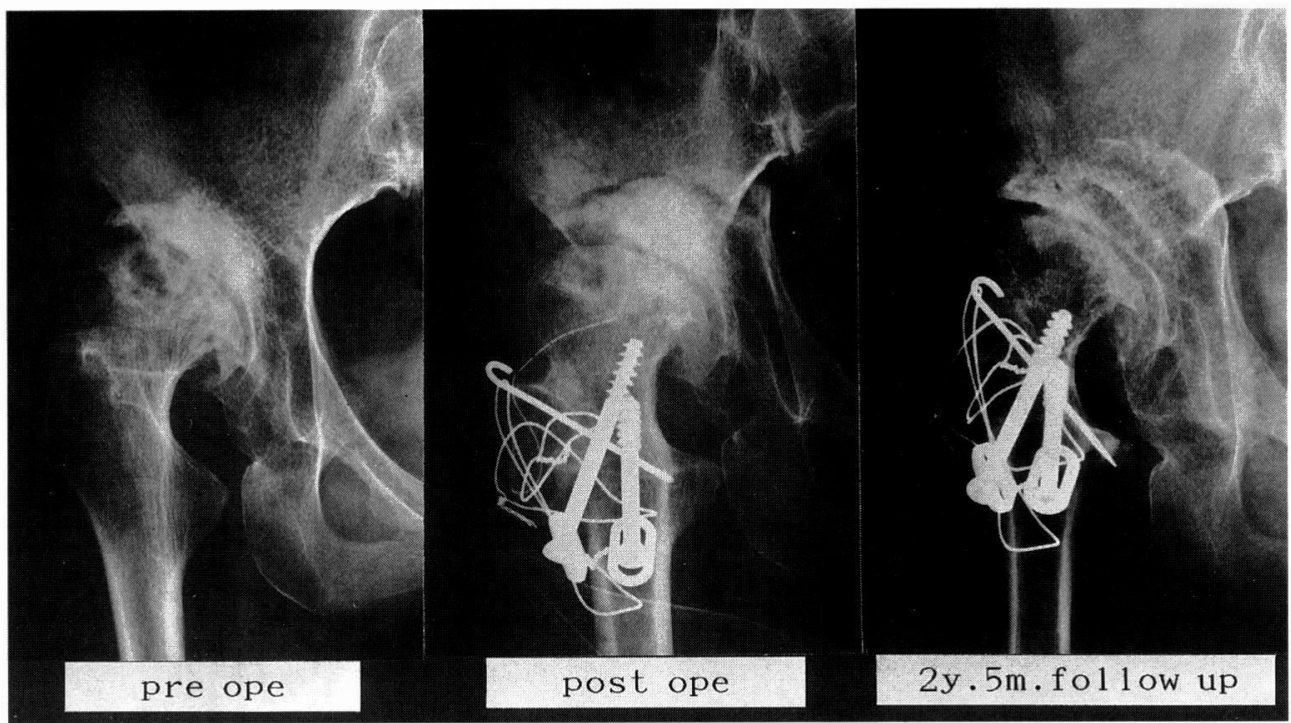

Fig. 4. Case 2. 40y. F. OA late stage, hypertrophic type: Neck $\%$ age matched $88 \%$, Chiari and valgus osteotomy. JOA score improved from 29 points to 91 points at 2 years 5 months follow up.

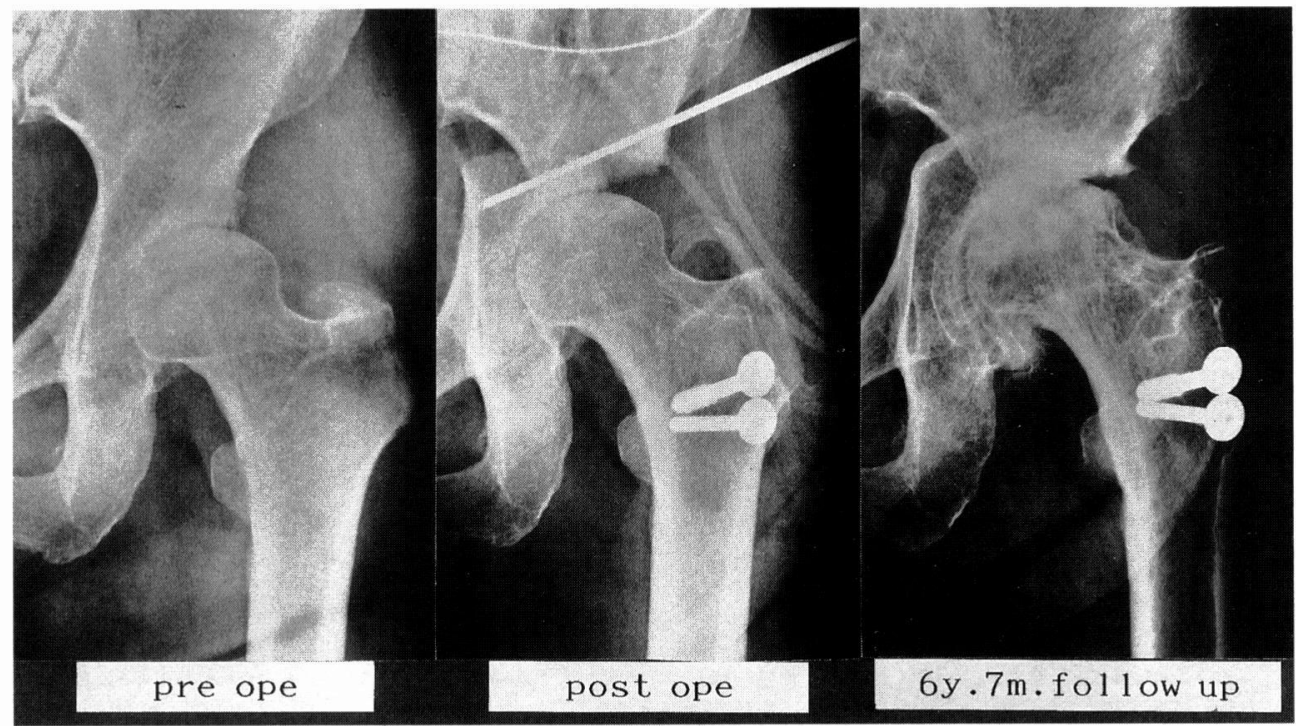

Fig. 5. Case 3. 56y. F. OA advanced stage, atrophic type: Neck\% age matched $149 \%$, total body $111 \%$, spine $139 \%$. Chiari pelvic osteotomy. JOA score decreased from 78 points to 68 points at 6 years 7 months follow up. 


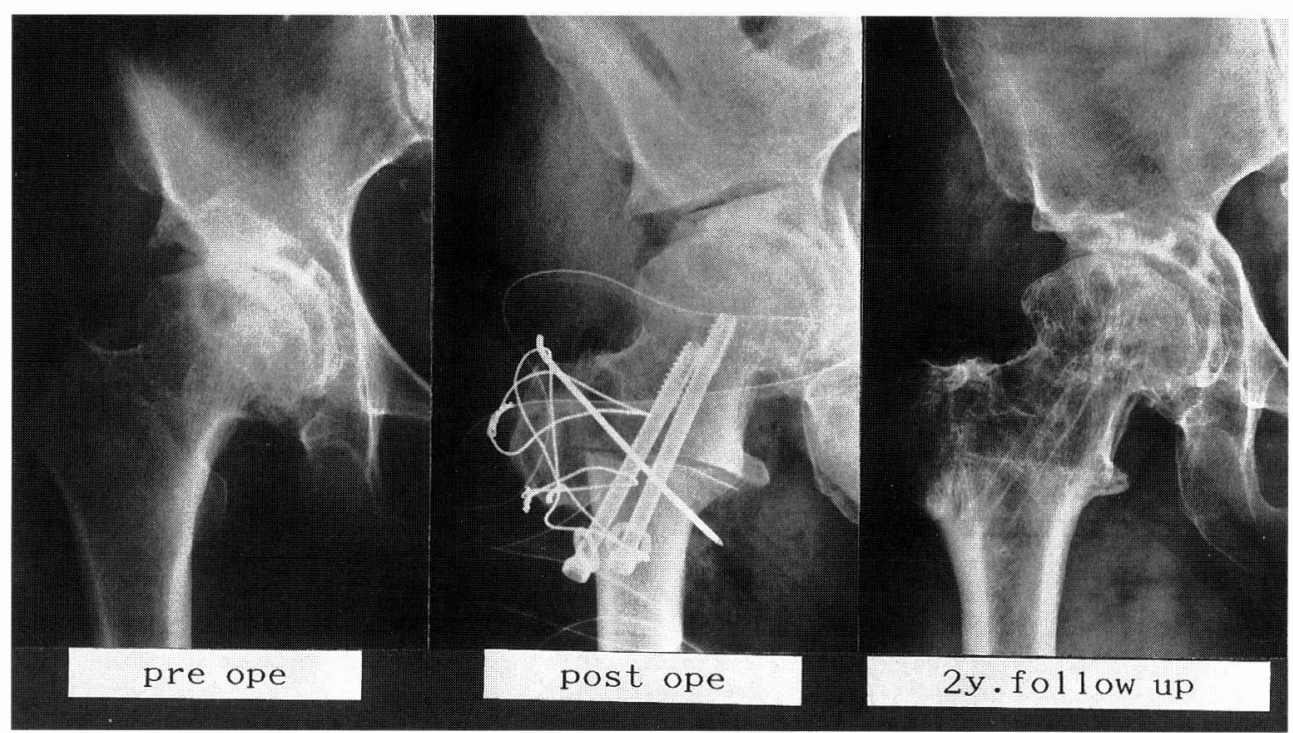

Fig. 6. Case 4. 56y. F. OA advanced stage, normotrophic type: Neck\%age matched $125 \%$, total body $94 \%$, spine $90 \%$. Chiari and valgus osteotomy. JOA score improved from 24 points to 89 points at 2 years follow up.

TABLE 2.

Comparison between Bombelli classification and \% age-matched BMD

\begin{tabular}{llrrr}
\hline Region & Bombelli & $\mathrm{n}$ & mean (\%) & s.d. \\
\hline \multirow{3}{*}{ Neck } & hypertrophic & 12 & 119.1 & 42.6 \\
& normotrophic & 14 & 123.5 & 28.1 \\
& atrophic & 9 & 123.2 & 65.5 \\
\hline \multirow{3}{*}{ Total body } & hypertrophic & 9 & 98.3 & 5.2 \\
& normotrophic & 9 & 103.0 & 12.4 \\
& atrophic & 4 & 106.0 & 10.7 \\
\multirow{3}{*}{ Spine } & hypertrophic & 9 & 105.2 & 18.5 \\
& normotrophic & 9 & 101.4 & 17.4 \\
& atrophic & 4 & 102.0 & 24.7 \\
\hline
\end{tabular}

The Bombelli classification was not correlated with systemic BMD.

improvement, and these degrees were then compared among the Bombelli types. In the hypertrophic type hips, the mean degree in improvement was $40 \pm$ 14.65 , and the patients' satisfaction was high (Figs 3 and 4). In the atrophic type hips, many patients were unsatisfied with the results (Fig. 5). There was a significant difference in the mean degree of improvement between these two groups (Fig. 1). This difference was especially significant in terms of pain (Fig. 2). In the normotorophic type hips, the degree of improvement tended to be higher than that in the atrophic type hips, but there was no significant difference between them (Fig. 6).

The \% age-matched values of the BMD of the hip joint, total body, and of the lumbar spine were compared among the three Bombelli types. There was no significant difference in the BMD of the femoral neck among the hypertrophic, normotrophic and atrophic types. The results were similar for the BMD for of Ward's triangle, and of the greater trochanter. Moreover, there was no significant difference in BMD of the total 
body or lumbar spine among the Bombelli types. That is, the Bombelli classification was not correlated with either local or systemic BMD (Table 2).

\section{Discussion}

Ohsawa et al. (1994) reported the postoperative results of a joint-preserving operation according to the Bombelli classification for $\mathrm{OA}$ in the advanced and late stage and reported that the results were worst in the atrophic type. And, that osteoid formation was less. in the atrophic type than in the hypertrophic type on histological examination, and suggested that it was an osteoblastic response that most influenced the regeneration of the articular cartilage and remodeling of the joint. The influence of a biological response on the postoperative results of THA has been discussed in recent years. Saito et al. (1987) stated similar results with the bony tissue of the acetabulum.

The improvements brought about by Chiari osteotomy are attributable at first to the decrease in stress due to the achieved medial displacement in the weight-bearing point, and next to the metaplasia of the joint capsule inserted between the femoral head and new acetabulum (Hiranuma et al. 1992). There have been several studies on the factors determining the postoperative outcome after Chiari operation. Inoue (1989) suggested that the remodeling factor was the major responsible factor for those aged up to their early forties, and that the age, preoperative stage, and displacement of the greater trochanter were the major factors for those near fifty or older. Other factors were determined to be the obtained congruity and the stability of the joint directly after the operation, and the strength of the bony acetabulum of the ilium.

The bone strength is supposed to be determined by the hardness and the elasticity of the bone, and the trabecular pattern. Since bone hardness comprises $80 \%$ of the strength, then the BMD is a major factor in the bone strength. In recent years DXA method has been frequently used for bone mineral measurement. The exposure dosage is slight in DXA and DXA is excellent in accuracy and precision. In this study, the BMD was measured in the femoral neck, Ward's triangle, and greater trochanter, as well as in the total body and the lumbar spine.

Between before and after the operation, the BMD showed a decrease in the lumbar spine in 6 cases but had no effect on the postoperative results. There was also no correlation between the BMD of each site and the clinical results. That is, the strength of the new bony acetabulum formed by Chiari operation was considered not to be influenced by the BMD of the hip, total body or lumbar spine.

Masuhara et al. (1994) concluded that the BMD of the hip joint was not related to the preoperative clinical evaluation and preoperative X-ray findings. Solomon and Schnitzler (1983) found that the osteoblastic change was due to local anatomical factors. In the present study, osteoblastic changes in OA were decided by the local surroundings at the joint regardless of bone changes in the total body. In conclusion 
the postoperative results after Chiari pelvic osteotomy for patients with osteoarthritis in the advanced and late stage were not correlated with bone mineral density. The postoperative results in patients with a hypertrophic type hip, in the Bombelli classification based on preoperative X-ray findings, were better than those in patients with an atrophic type. I postulate that local biological response was involved in the postoperative results after Chiari pelvic osteotomy.

Acknowledgments: The author thanks Professor Akio Inoue and Dr. Fujio Higuchi, (Department of Orthopaedic Surgery, Kurume University School of Medicine) for their kind advice.

\section{References}

Bombelli R. Osteoarthritis of the hip: classification and pathogenesis. 2nd edn. SpringerVerlag, Berlin Heidelberg New York, 1983.

Hiranuma S, Higuchi F, Inoue A, and Miyazaki $M$. Changes in the interposed capsule after
Chiari osteotomy: an experimental study on rabbits with acetabular dysplasia. J Bone Joint Surg 1992; 74-B:463-467.

Inoue A. Pelvic osteotomies in the treatment of coxarthrosis -with special reference to Chiari pelvic osteotomy-. J Jpn Orthop Assoc 1989; 63:1587-1598.

Inoue A, Higuchi F, and Shiba N. Chiari pelvic osteotomy for coxarthrosis in adults. J Orthop Surg Tech 1990; 5:105-111.

Masuhara K, Kato Y, Ejima Y, Fuji T, and Hamada $\mathrm{H}$. Bone mineral assessment by dualenergy X-ray absorptiometry in patients with coxarthrosis. International Orthopaedics (SICOT). 1994; 18:215-219.

Ohsawa S, Matsushita S, and Ueno R. Jointpreserving operations for elderly patients with advanced and late-stage coxarthritis. Arch Orthop Trauma Surg 1994; 113:302-307.

Saito M, Saito S, Ohzono K, and Ono K. The osteoblastic response to osteoarthritis of the hip: Its influence on the long-term results of arthroplasty. J Bone Joint Surg 1987; 69B:746-751.

Solomon L, and Schnitzler CM. Pathogenetic type of coxarthrosis and implications for treatment. Arch Orthop Trauma Surg 1983; 101:259-261. 